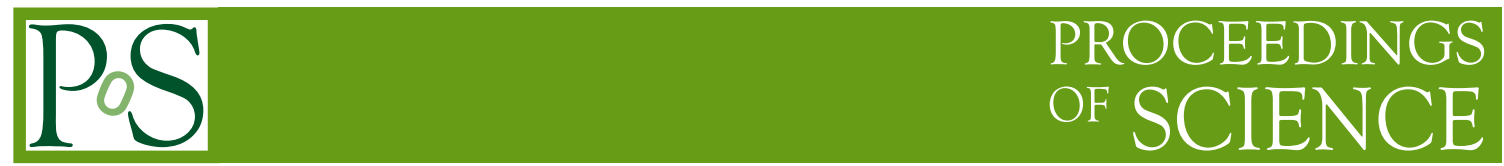

\title{
Higgs Beyond the Standard Model at the LHC
}

\author{
Marta Felcini ${ }^{* \dagger}$ \\ University College Dublin \\ E-mail: marta.felcini@cern.ch
}

\begin{abstract}
We present an overview of the strategies devised by the ATLAS and CMS experiments to search and discover Higgs bosons beyond the Standard Model. Once one or more Higgs-like signals will have been observed, detailed measurements will provide further insight on the nature of the observed signals.
\end{abstract}

XXth Hadron Collider Physics Symposium

November 16 - 20, 2009

Evian, France

\footnotetext{
*Speaker.

${ }^{\dagger}$ On behalf of the ATLAS and CMS Collaborations.
} 


\section{Introduction}

The standard model (SM) of elementary particle physics is successfully explaining all observed fundamental interactions at energy scales up to few hundreds $\mathrm{GeV}$, as studied in the last decades with experiments at high energy electron-positron and hadron colliders. Within the framework of the SM, one Higgs boson is expected to exist as a result of the electroweak symmetry breaking, needed to provide mass to the heavy electroweak $\mathrm{W}$ and $\mathrm{Z}$ bosons and to the matter fermions (quarks and leptons). In spite of extensive searches, there is to date no direct evidence of the existence of the SM Higgs boson. The SM Higgs search strategies at colliders [1] are based on Higgs production and decay properties, precisely predicted by the SM, as a function of the Higgs mass. The SM does not predict the value of the Higgs mass, but rather requires that, for the theory to be perturbative up to a scale of new physics $\Lambda$, the Higgs mass be significantly lower than one $\mathrm{TeV}$, depending on the scale of new physics. As an example, for $\Lambda$ at the GUT scale $\left(O\left(10^{16}\right) \mathrm{GeV}\right)$ the Higgs boson is expected to be lighter than $\sim 200 \mathrm{GeV}$, while for $\Lambda \sim 1 \mathrm{TeV}$ an upper bound of $\sim 700 \mathrm{GelV}$ is derived [2]. Based on the experimental limit from direct searches and from fits to precision electroweak measurements, the preferred (95\% CL) SM Higgs mass range lies between the LEP lower limit of $114.4 \mathrm{GeV} \mathrm{[3]} \mathrm{and} \mathrm{the} \mathrm{upper} \mathrm{limit} \mathrm{from} \mathrm{electroweak} \mathrm{fits,} \mathrm{presently} 157 \mathrm{GeV}$ (not including the direct LEP Higgs mass lower limit) [4].

Notwithstanding its successes, the SM is considered to be an effective theory, that cannot provide solutions to several outstanding problems. TeV scale supersymmetry is one of the most popular extensions of the SM. Supersymmetry solves the problem of quadratically divergent corrections to the Higgs boson mass by the introduction of new bosonic particles, having the same couplings as the SM fermions, and of new fermions, having the same couplings as the SM bosons, thus canceling the loop contributions to the Higgs mass to all orders. The minimal supersymmetric standard model (MSSM) represents the minimal particle content for a supersymmetric extension of the SM. Other possible solutions to the hierarchy problem include the five-dimensional RandallSundrum model [5], in which all mass terms near the Planck mass are suppressed by an exponential factor, bringing them down to the $\mathrm{TeV}$ scale, and the Littlest Higgs model [6], in which a SM-like Higgs boson remains light and a small set of new heavy particles (heavy gauge bosons, $W^{\prime}, Z^{\prime}$. a vector-like heavy T-quark pair and a pair of doubly-charged Higgs bosons) is introduced, canceling out the most significant loop corrections to the SM-like Higgs mass. Scenarios beyond the SM (BSM) may predict the existence of several Higgs particles. Their properties (such as mass, spin, S, charge, C, parity, P, couplings) are depending on the specific model. Specific BSM models are needed as benchmarks to prepare the experimental search strategy, notably the trigger selections, ensuring that potentially interesting signals are detected and recorded on-line with maximal efficiency. Differently from the SM, where Higgs couplings to fermions and bosons are precisely predicted, in BSM scenarios Higgs couplings are model-dependent. In addition to the Higgs mechanism, other models have been proposed that use composite Higgs bosons and strongly interacting dynamics to break the electroweak symmetry. Possible signatures would be Higgs production with anomalous Higgs couplings and an enhanced vector boson pair $(V V, V=W, Z)$ production crosssection [7]. Thus, beyond early discoveries of Higgs-like signals, precise measurements of their properties are essential for an in-depth understanding of the underlying physics. 


\begin{tabular}{lcl}
\hline $\begin{array}{l}\text { Possible outcome of experimental searches: } \\
\text { direct observation and early mass measurements }\end{array}$ & $\begin{array}{c}\text { Consistent } \\
\text { with SM? }\end{array}$ & $\begin{array}{l}\text { Open questions } \\
\text { Need additional measurements }\end{array}$ \\
\hline Only one neutral low mass Higgs & Yes & SM, MSSM, other BSM? \\
Only one neutral high mass Higgs & No & MSSM, other BSM ? \\
More than one neutral Higgs & No & MSSM, other BSM ? \\
Charged Higgs & No & MSSM, other BSM ? \\
No Higgs, other BSM particles/interactions & No & BSM Model? EWSB mechanism? \\
No Higgs, no other BSM particles/interactions & No & BSM Model? EWSB mechanism? \\
\hline
\end{tabular}

Figure 1: Overview of possible outcomes of the Higgs searches and open questions to be investigated.

\section{Preparation for BSM Higgs discoveries at the LHC}

The experiments at the Large Hadron Collider (LHC) [8] have the primary goal of discovering at least one Higgs boson, as well as searching for signals of physics beyond the SM at the TeV scale. The LHC is designed to collide proton beams, up to an energy of $7 \mathrm{TeV}$ per beam, every $25 \mathrm{~ns}$, with an instantaneous luminosity up to $10^{34} \mathrm{~cm}^{-2} \mathrm{~s}^{-1}$, making it the highest energy and luminosity proton-proton collider ever built. Two general-purpose detectors, ATLAS and CMS, are constructed to record and analyze the results of these collisions. Both the collider and the detectors have started successful operation with first proton-proton collision data-taking in November 2009 , with a collision center-of-mass energy, $\sqrt{s}$, up to $2.36 \mathrm{TeV}$. The LHC and the detectors have resumed collision data-taking on March 30th, 2010, at a collision energy of $7 \mathrm{TeV}$. Continuous operation at this collision energy is planned to last several months in view of collecting an integrated luminosity of at least one $\mathrm{fb}^{-1}$. The design collision energy of $14 \mathrm{TeV}$ will be reached in 2013 . Detailed simulation studies of Higgs discovery reach have been reported so far at $\sqrt{s}=14 \mathrm{TeV}$ or $10 \mathrm{TeV}$. Estimations for the Higgs discovery reach at $\sqrt{s}=7 \mathrm{TeV}$ are in progress. An example is given in Section 3.

The main goal of the BSM Higgs studies carried out so far with simulated (MC) data is to setup the search strategies - trigger conditions, background estimation methods, analysis tools - into final states and topologies different from those studied for the SM Higgs searches, but potentially relevant and even dominant in BSM scenarios. Final states with multiple b quarks and/or $\tau$ leptons, lepton resonances, cascade decays through, e.g. heavy supersymmetric particles, invisible decays into e.g. neutralinos, can be dominant Higgs signatures in BSM scenarios. ATLAS and CMS MC studies result into predictions of the experimental discovery reach. First observations (with early measurements of mass, charge, etc.) and detailed measurements (couplings, spin, CP values) are needed to disentangle among different hypothesis and determine the role of the newly detected Higgs-like particles.

The main steps in preparation for the search are:

- determination of trigger conditions to be highly efficient on BSM look-alike SM signatures (in loosely defined signal regions, that are not strictly dependent on specific model assumptions); 
- estimation of SM background contributions (W/Z+jets, $t \bar{t}+$ jets, WW, WZ, ZZ,etc.) and definition of reliable methods to measure and validate the SM background predictions;

- definition of specific signal regions (model dependent) and determination of discriminating quantities to maximize signal-to-background ratio, while minimizing measurement (statistical and systematic) uncertainties;

- maximization of the discovery reach with early measurements (observables: signal event yield, mass, charge), as a function of model parameters, depending on integrated luminosity;

- in view of different possible outcomes of the experimental searches (see Figure 1), definition of methods for detailed measurements, to determine the role of the newly detected particles and the underlying physics.

The results of these studies at $14 \mathrm{TeV}$ are extensively documented in the ATLAS [11] and in the CMS [12] reports. An overview of these results is given in the following.

\section{Expectations for Higgs signal discovery in the MSSM}

The MSSM requires two Higgs doublets, resulting into five physical Higgs (spin 0) bosons: a light, neutral, $\mathrm{CP}$-even, $\mathrm{h}$, a heavier neutral, $\mathrm{CP}$-even, $\mathrm{H}$, a neutral, $\mathrm{CP}$-odd, $\mathrm{A}$, and two charged $H^{ \pm}(\mathrm{C}= \pm 1)$ Higgs bosons. They decay to known bosons and fermions, with branching ratios that depend on the model parameters. MSSM Higgs bosons may also decay into SUSY particles, if kinematically accessible, resulting into visible or invisible cascade decays. At tree level, two model parameters, usually chosen to be the A mass, $M_{A}$, and the ratio of the vacuum expectation values of the two Higgs doublets, $\tan \beta$, determine the Higgs masses and couplings. Radiative corrections, mainly from top and scalar top masses, at small $\tan \beta$, or from bottom and scalar bottom masses, at large $\tan \beta$, introduce further model parameter dependence. Including radiative corrections, and irrespective of model parameter values, a theoretical upper (lower) limit $m_{h}^{\max } \simeq 135 \mathrm{GeV}$ is set on the lighter (heavier) CP-even Higgs mass $m_{h}\left(M_{H}\right)$. Additional predictions [9] are that for large $M_{A}$ values $\left(M_{A} \gtrsim m_{h}^{\max }\right)$, then $M_{H} \sim M_{A}$, while $m_{h}$ reaches its upper bound and the lightest Higgs phenomenology becomes SM-like. In the small $M_{A}$ range, $M_{A} \lesssim m_{h}^{\max }$ and $M_{H} \gtrsim m_{h}^{\max }$, while $m_{h} \sim M_{A}$. The charged Higgs mass $M_{H^{ \pm}}$is expected to be larger than the $\mathrm{W}$ mass, and larger than $M_{A}$, irrespective of $\tan \beta$. The present experimental status of the searches for neutral and charged Higgs bosons has been reviewed at this Symposium [10] and summarized in terms of excluded regions in the $\left(M_{A}, \tan \beta\right)$ plane. From LEP Higgs searches, the region $0.7 \lesssim \tan \beta \lesssim 2$, is excluded, irrespective of $M_{A}$, while $m_{h}\left(M_{A}\right) \lesssim 93 \mathrm{GeV}$ is excluded irrespective of $\tan \beta$. From Tevatron Higgs searches, a region for $\tan \beta \gtrsim 40$ to 50 , depending on $M_{A}$, is excluded in the range $M_{A} \lesssim 200$ $\mathrm{GeV}$. The precise limit values depend on the top mass $M_{t}$ and on the SUSY breaking mass scale. Figure 2 shows an overview of neutral and charged Higgs bosons production processes, decays and final states, studied to establish MSSM Higgs search strategies at the LHC. For neutral MSSM Higgs bosons, the dominant production mechanism is the gluon fusion processes $g g \rightarrow h, H, A$, for small and moderate values of $\tan \beta$. The cross-section is expected to be higher than in the SM because of additional heavy particles contributing to the loops. The vector boson fusion (VBF) process $p p \rightarrow q q+W W / Z Z \rightarrow q q+h / H$ plays an important role for the light scalar $h$, close to its 


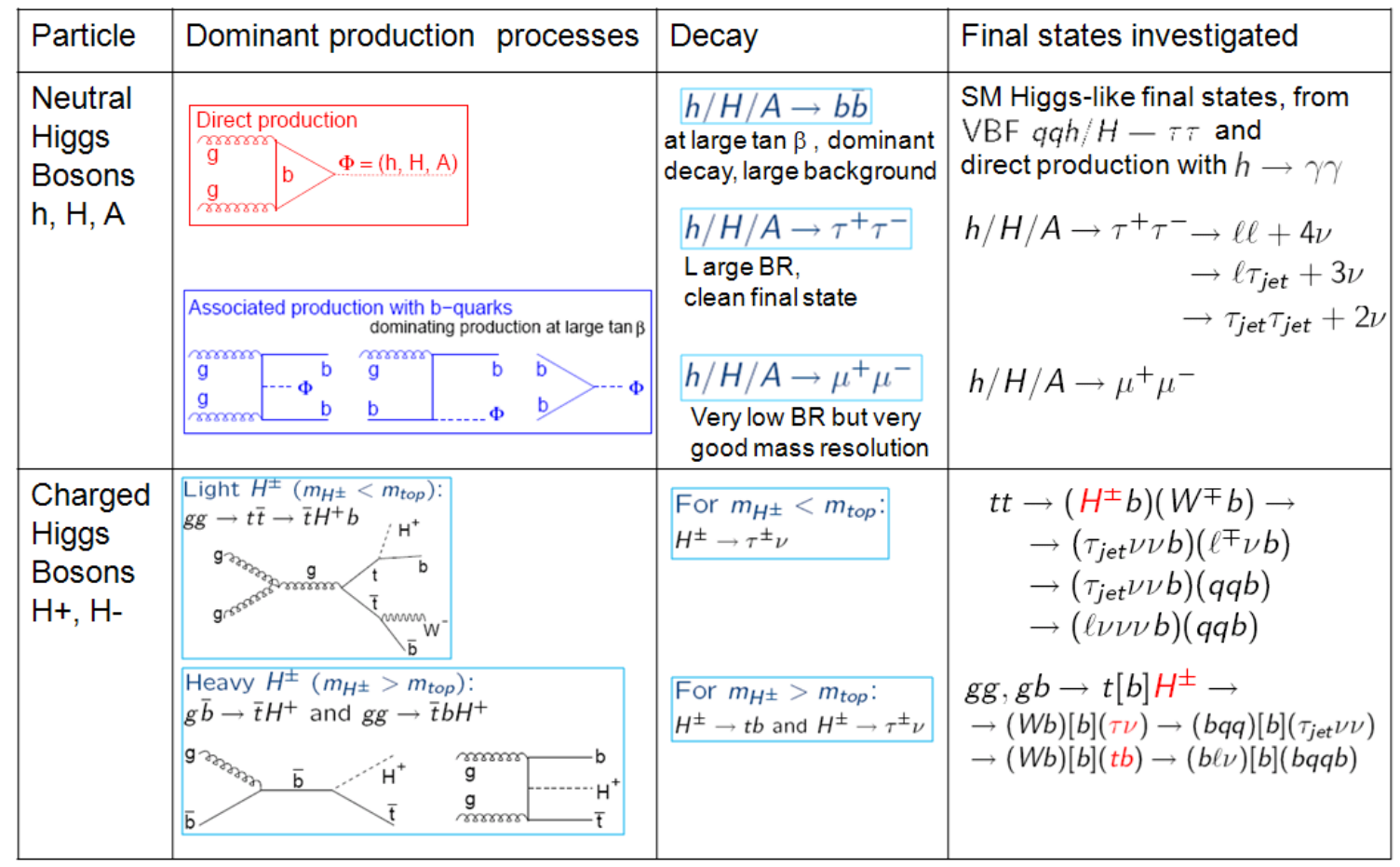

Figure 2: Overview of neutral and charged Higgs bosons production processes, decays and final states, studied to establish MSSM Higgs search strategies at the LHC.

upper mass bound, where it becomes SM-like, and for the heavy scalar $H$ near its lower mass bound $\left(m_{h}^{\max }\right)$. For large values of $\tan \beta$, Higgs associated productions with bottom quarks (Figure 2) are the dominant production mechanisms, with negligible contributions from other processes. For large values of $\tan \beta$, the down (up)-type Yukawa couplings are strongly enhanced (suppressed), apart from the region where the light (heavy) scalar is near its upper (lower) mass bound. As the main decay mode $h / H / A \rightarrow b b$ suffers from high QCD background, the decay $h / H / A \rightarrow \tau^{+} \tau^{-}$, with all possible $\tau^{+} \tau^{-}$final states (jet jet, e jet, $\mu$ jet and e $\mu$ ), offers a cleaner signature, reaching about $10 \%$ branching ratio for $\tan \beta>10$. In the large $\tan \beta$ and small $M_{A}$ region, the $h, H, A \rightarrow \mu \mu$ decay is also exploited. In spite of the small expected branching ratio $\left(\sim 10^{-4}\right)$, it offers the possibility to fully reconstruct the Higgs mass with superior mass resolution. In contrast to the SM, the total widths of the MSSM Higgs bosons do not exceed few tens of GeV, so that the MSSM Higgs particles appear as narrow resonances. Figure 3 summarizes the LHC prospects for MSSM neutral Higgs searches and discoveries, in terms of the regions of the $\left(M_{A}, \tan \beta\right)$ plane where an estimated $5 \sigma$ signal significance or a $95 \%$ signal exclusion can be established at the LHC. The CMS results presented here are based on full detector simulation, considering trigger conditions for low instantaneous luminosity $\left(L=2 \cdot 10^{33} \mathrm{~cm}^{-2} \mathrm{~s}^{-1}\right)$. The expected systematic uncertainties are included in the evaluation of the signal significance. Similar results are reported by the ATLAS Collaboration. The discovery or exclusion regions are shown in the $m_{h}^{\max }$ scenario, designed to provide the most conservative MSSM exclusion limits at LEP.

The discovery of a charged Higgs boson $H^{ \pm}$would be direct evidence of physics beyond the 


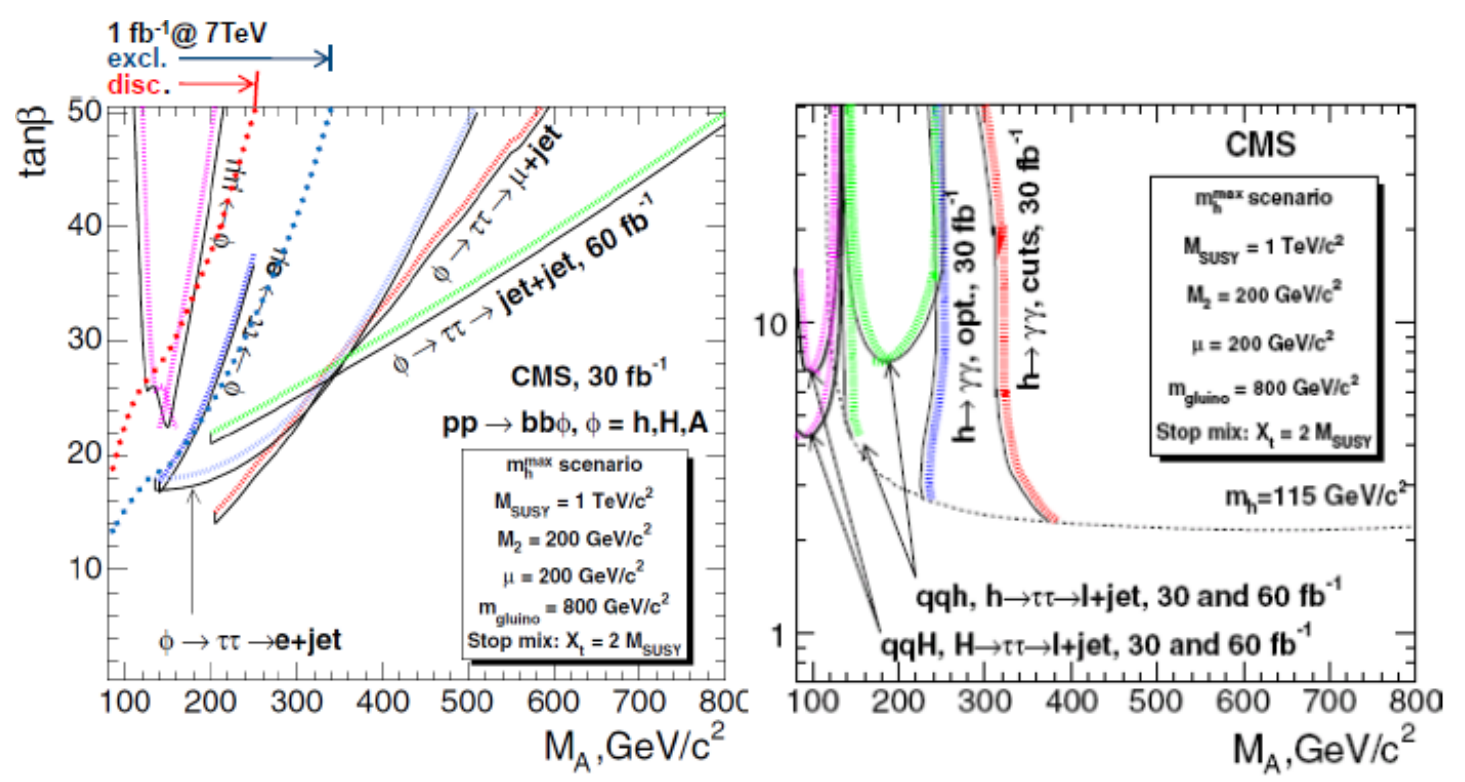

Figure 3: Overview of the CMS discovery reach for MSSM neutral Higgs bosons. The left panel shows (hatched) the regions covered by the search at $14 \mathrm{TeV}$ [12] for MSSM associate $b b \Phi$ production $(\Phi=$ $h, H, A)$ and $\Phi \rightarrow \tau_{j e t} \tau_{j e t}, \tau_{j e t} \tau_{e, \mu}, \tau_{e} \tau_{\mu}$. The superimposed red and blue dotted lines, on the left panel, are the boundaries of, respectively, the projected discovery and exclusion regions [13], to be covered by the combined search for $b b \Phi$ and $\Phi \rightarrow \tau_{j e t} \tau_{j e t}, \tau_{j e t} \tau_{e, \mu}, \tau_{e} \tau_{\mu}$, with $1 \mathrm{pb}^{-1}$ of data at a collision energy of $7 \mathrm{TeV}$ (expected to be delivered by the LHC in the 2010-2011 run). The right panel shows the discovery regions at $14 \mathrm{TeV}$ [12] of the searches for MSSM $b b h$ and $h \rightarrow \gamma \gamma$, or $q q h / H$ and $h / H \rightarrow \tau_{j e t} \tau_{e, \mu}$. The latter is exploited to cover the difficult region at intermediate $\tan \beta$ and low Higgs mass values. The large $M_{A}$ and $\tan \beta$ region is covered by the SM-like $h \rightarrow \gamma \gamma$ search.

SM. The search strategies depend on the value of the $H^{ \pm}$mass, $M_{H^{ \pm}}$, relative to the mass of the top quark, $M_{t}$. Charged Higgs bosons couple to fermions as $H^{ \pm} f_{u} f_{d} \sim m_{f_{d}} \tan \beta+m_{f_{u}} / \tan \beta$. They are produced (Figure 3) in top quark decay, via the $t \bar{t}$ process, if $M_{H^{ \pm}}<M_{t}$, or in association with a top quark, through gluon-bottom fusion $\left(g b \rightarrow H^{ \pm} t\right)$, if $M_{H^{ \pm}}>M_{t}$. Light charged Higgs bosons decay almost exclusively to $\tau \nu$ for $\tan \beta \gtrsim 3$. For $M_{H^{ \pm}}>M_{t}+m_{b}$, the decay $H^{ \pm} \rightarrow t b$, dominates with an important contribution of the leptonic decay $H^{ \pm} \rightarrow \tau \nu$ at large $\tan \beta$. The leptonic decay signature is used for the search, because of the lower background. For a light charged Higgs boson, produced through $t \bar{t} \rightarrow H^{ \pm} b W b$, three different signal final states have been studied (see Figure3). The main background comes from $t \bar{t}$ events, with significant contribution from $\mathrm{W}+$ jets events. In the heavy charged Higgs search, with $g g, g b \rightarrow t[b] H^{ \pm}$, two final states have been analyzed, with $t[b] H^{ \pm} \rightarrow b q q[b] \tau \nu$ or $\rightarrow b \ell v[b] b q q b$. In the former case, the reconstruction of the top quark mass is essential to the signal identification. The charged Higgs mass is then estimated by the transverse mass $M_{T}=M_{T}\left(\tau, E_{T}^{\text {miss }}\right)$ system. By requiring $M_{T}>100 \mathrm{GeV}$, a clean signal selection is achieved. Figure 4 shows the expected results of the ATLAS heavy charged Higgs search with an integrated luminosity of 1,10 and $30 \mathrm{fb}^{-1}$. 

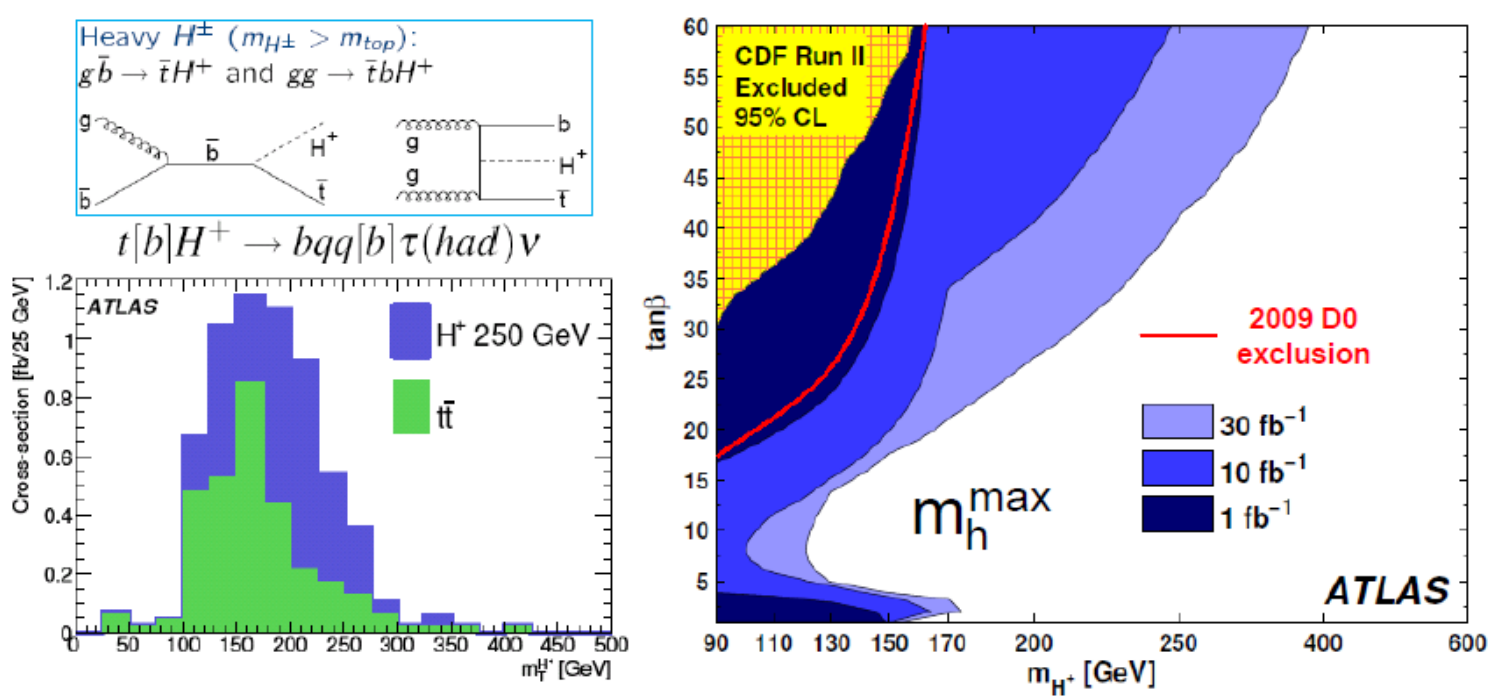

Figure 4: ATLAS charged Higgs search [11]. Left: heavy charged Higgs production, sought final state and expected signal (for $M_{H^{ \pm}}=250 \mathrm{GeV}$ ) plus background distribution of the reconstructed charged Higgs mass. Right: regions of the $\left(M_{H^{ \pm}}, \tan \beta\right)$ plane where a signal significance of at least $5 \sigma$ can be observed, with an integrated luminosity of 1,10 and $30 \mathrm{fb}^{-1}$. The superimposed red curve shows the region excluded by Tevatron searches at $95 \%$ CL.

\section{Expectations for Higgs signal discovery beyond the MSSM}

Beyond the MSSM, several scenarios are possible, as mentioned in Section 1. Hereafter, we recall two examples of Higgs searches in BSM scenarios other than the MSSM. For a more complete discussion of the search strategies in various BSM scenarios, we refer to the original literature $[11,12]$ and references therein.

The first example refers to invisible Higgs decays. If a Higgs boson decays into undetectable particles (like in models predicting weakly interacting stable particles. e.g. neutralinos, gravitinos, gravitons or Majorons). then the primary question is whether it is possible to trigger on events with Higgs production and undetectable decays. The ATLAS studies [11] show that effective signal triggering, with tolerable background trigger rates, is possible in the case of Higgs production via vector boson fusion (VBF) or via $\mathrm{HZ}$ associate production (see Figure 5), requiring a forward jet plus a central jet plus large missing transverse energy, in the first case, or a di-lepton pair in the second case. Beyond trigger strategies, complete analysis methods have also been developed based on sequential cuts, for the VBF Higgs production channel, or multivariate analysis methods, such as boosted decision trees (BDT), for the $\mathrm{HZ}$ associate production. It is found that the VBF analysis performs better than the $\mathrm{ZH}$ analysis for high Higgs masses. However, in case of observation of an invisible Higgs-like signal in the VBF channel, the $\mathrm{HZ}$ analysis will help to establish the nature of the observed signal as stemming from Higgs production and invisible decay. The projected results are summarized in Figure 5, as excluded regions in the plane $\left(M_{H}, \xi^{2}\right)$. The parameter $\xi^{2}$ is defined as the ratio between the BSM and SM Higgs production cross-section, times the invisible Higgs decay branching ratio, $\xi^{2}=\mathrm{B}(H \rightarrow i n v) \sigma_{B S M} / \sigma_{S M}$. An invisible Higgs signal can 


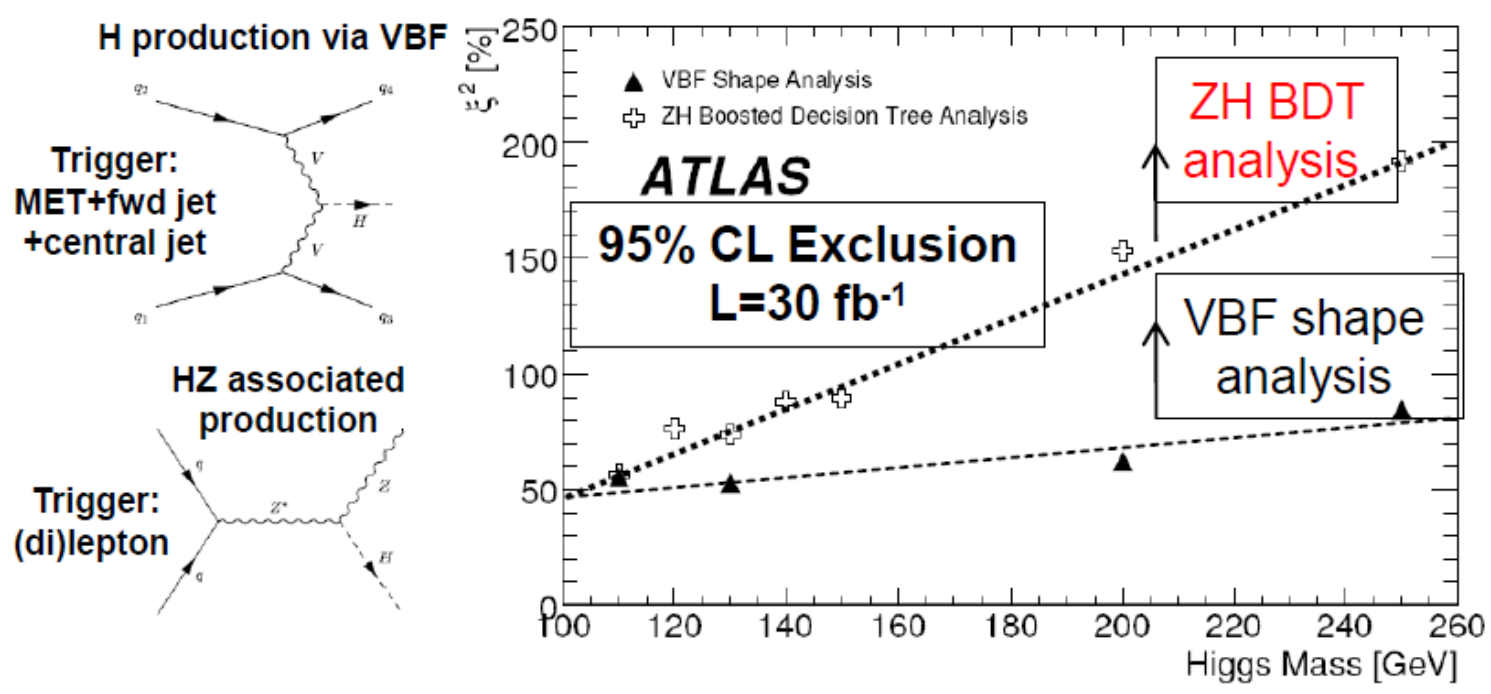

Figure 5: ATLAS invisible Higgs search [11]: the Higgs production processes exploited for the search are displayed together with the trigger conditions used for the on-line selection of the events. On the right, the excluded regions in the plane $\xi^{2}$-vs- Higgs mass are shown for the two analyses (VBF shape analysis and ZH BDT analysis) employed for the search.

be excluded (observed) with $30 \mathrm{fb}^{-1}\left(\sim 70 \mathrm{fb}^{-1}\right)$ for $\xi^{2} \geq 0.5$, such as e.g. for $\sigma_{B S M} \geq 0.5 \sigma_{S M}$ and $\mathrm{B}(H \rightarrow i n v)=1$. Results from a similar study performed by the CMS Collaboration have been reported at this Symposium [15]

The second example of searches in models other than the MSSM refers to Higgs production and decays in the framework of an extra-dimension model, for example the five dimensional (5D) Randall-Sundrum (RS) model [5]. Like other extra-dimension models, it tries to relate two fundamental scales of physics, namely the Plank scale and the electroweak scale. In its simplest version, the RS model is based on a 5D space described by the metric $d s^{2}=e^{-2 k y} \eta_{\mu \nu} d x^{\mu} d x^{\nu}-d y^{2}$, where $\mathrm{k}$ is the 5D curvature parameter of order the Planck scale. In this space, two four-dimensional branes are separated by the distance $r_{c}$. The hierarchy problem can be solved if $r_{c}$ is such that $k r_{c}$ is of order 10. By placing all the SMl fields on the visible 4D brane, all the mass terms, which are of order the Planck mass, are rescaled by an exponential factor, $e^{-2 k r_{c} \pi}$, to a mass scale of order $1 \mathrm{TeV}$. The quantum excitations of the brane separation can be described in terms of a scalar field, the radion, which in general mixes with the Higgs boson. The scalar sector of the RS model is parameterized in terms of a dimensionless Higgs-radion mixing parameter $\xi$, the Higgs and radion masses, $m_{h}$, $m_{\phi}$, as well as the vacuum expectation value of the radion field, $\Lambda_{\phi}$. The phenomenology of Higgs and radion particles at LHC has been subject to several studies concentrating mainly on processes involving both types of particles. Indeed, the presence in the Higgs-radion sector of trilinear terms opens the possibility of $\phi \rightarrow h h$ and $h \rightarrow \phi \phi$ decays. For example, for $m_{h}=120 \mathrm{GeV}, \Lambda_{\phi}=5 \mathrm{TeV}$ and $m_{\phi} \sim 250-350 \mathrm{GeV}$ the branching ratio $B(\phi \rightarrow h h)$ ranges between 20 and $30 \%$.

The CMS discovery potential is estimated for the decay $\phi \rightarrow h h$, with $\gamma \gamma b \bar{b}, \tau \tau b \bar{b}$ and $b \bar{b} b \bar{b}$ final states and for an integrated luminosity of $30 \mathrm{fb}^{-1}$. The study has been carried out for $m_{\phi}=300$ $\mathrm{GeV}$ and $m_{h}=125 \mathrm{GeV}$. The sensitivity is evaluated in the $\left(\Lambda_{\phi}, \xi\right)$ plane, including systematics 

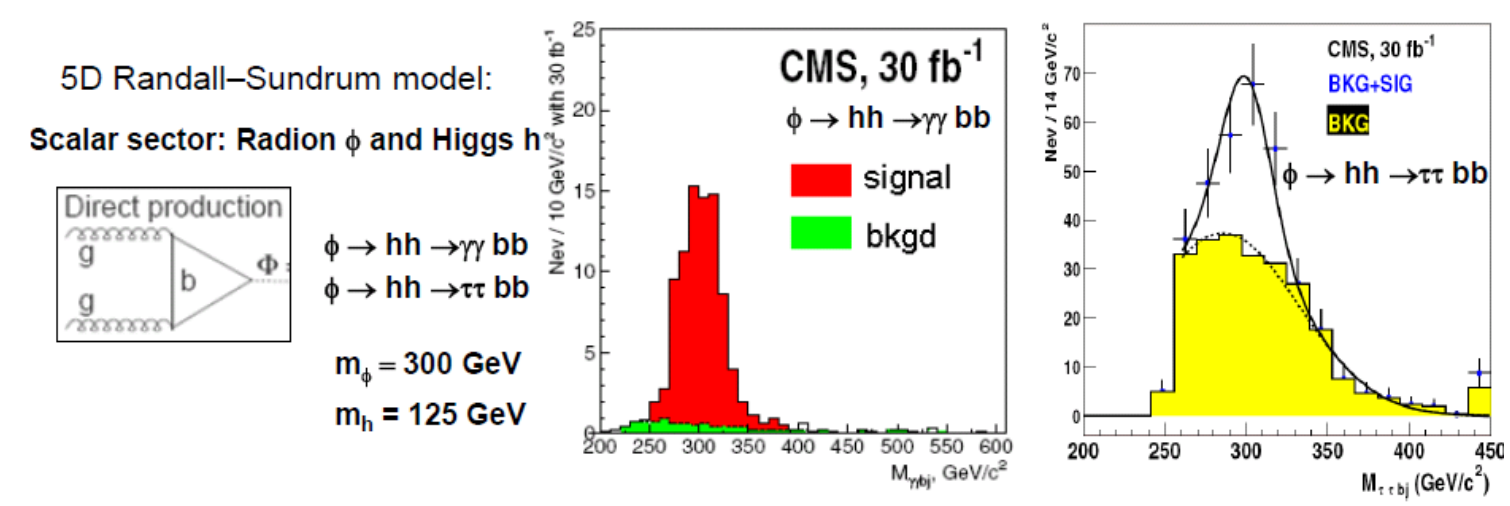

\section{RS scalar sector parameters:}

- Higgs - radion mix. parameter $\xi$

- radion mass $m_{\phi}$

- Higgs mass $m_{h}$

- v.e.v of the radion field $\Lambda_{\phi}$.
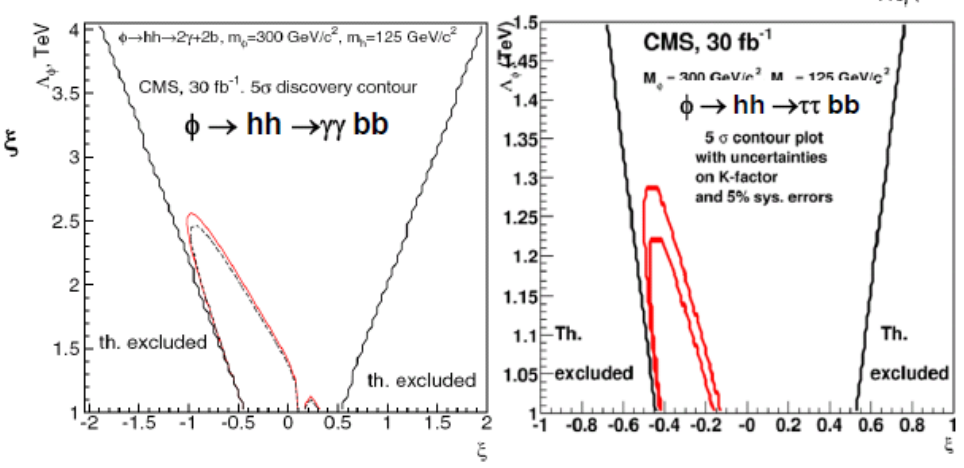

Figure 6: CMS search for the decay of a radion $\phi$ into a pair of Higgs bosons, in the $\phi \rightarrow \mathrm{hh} \rightarrow \gamma \gamma b \bar{b}$ and $\phi \rightarrow h h \rightarrow \tau \tau b \bar{b}$ channels. The radion production and decay processes are displayed together with the parameters of the scalar sector in a minimal 5D RS model. The reconstructed mass distributions in the $\gamma \gamma b j$ and $\tau \tau b j$ final states, after selection, are shown for background and signal, with maximal cross section times branching ratios value in the $\left(\Lambda_{\phi}, \xi\right)$ plane, together with the resulting $5 \sigma$ discovery contours with 30 $\mathrm{fb}^{-1}$ for the $\phi \rightarrow h h \rightarrow \gamma \gamma b \bar{b}$ and for the $\phi \rightarrow h h \rightarrow \tau \tau b \bar{b}$ channels. The solid (dashed) contour shows the discovery region without (with) the effects of the systematic uncertainties [12].

uncertainties. In Figure 6, the reconstructed mass $M_{\gamma \gamma b j}$ and $M_{\tau \tau b j}$ distributions for background and signal are shown together with the $5 \sigma$ discovery contours accessible with $30 \mathrm{fb}^{-1}$ for the $\phi \rightarrow h h \rightarrow \gamma \gamma b \bar{b}$ and $\phi \rightarrow h h \rightarrow \tau \tau b \bar{b}$ channels.

\section{Expectations for measurements of Higgs properties}

Beyond initial observations of Higgs-like signals, the LHC experiments will measure Higgs properties, such as masses, widths and couplings, spin and CP quantum numbers to underpin the fundamental role of the newly observed particles. Because of the high resolution with which leptons can be measured, Higgs final states with leptons are likely to provide the most precise measurements. In the following we show two examples of Higgs measurements in leptonic final states.

In supersymmetric scenarios, the Higgs boson production in association with b quarks, $p p \rightarrow$ $b \bar{b} \phi(\phi=h, H, A)$ followed by the $\phi \rightarrow \mu \mu$ decay can provide the best measurement for the mass and width of the heavy Higgs bosons $\mathrm{H}$ and $\mathrm{A}$. At high $\tan \beta$, the intrinsic Higgs width, sensitive to the $\tan \beta$ value, is comparable or larger than the dimuon mass experimental resolution, thus the measured width can be used to estimate accurately the $\tan \beta$ value. In spite of the small $\phi \rightarrow \mu \mu$ 

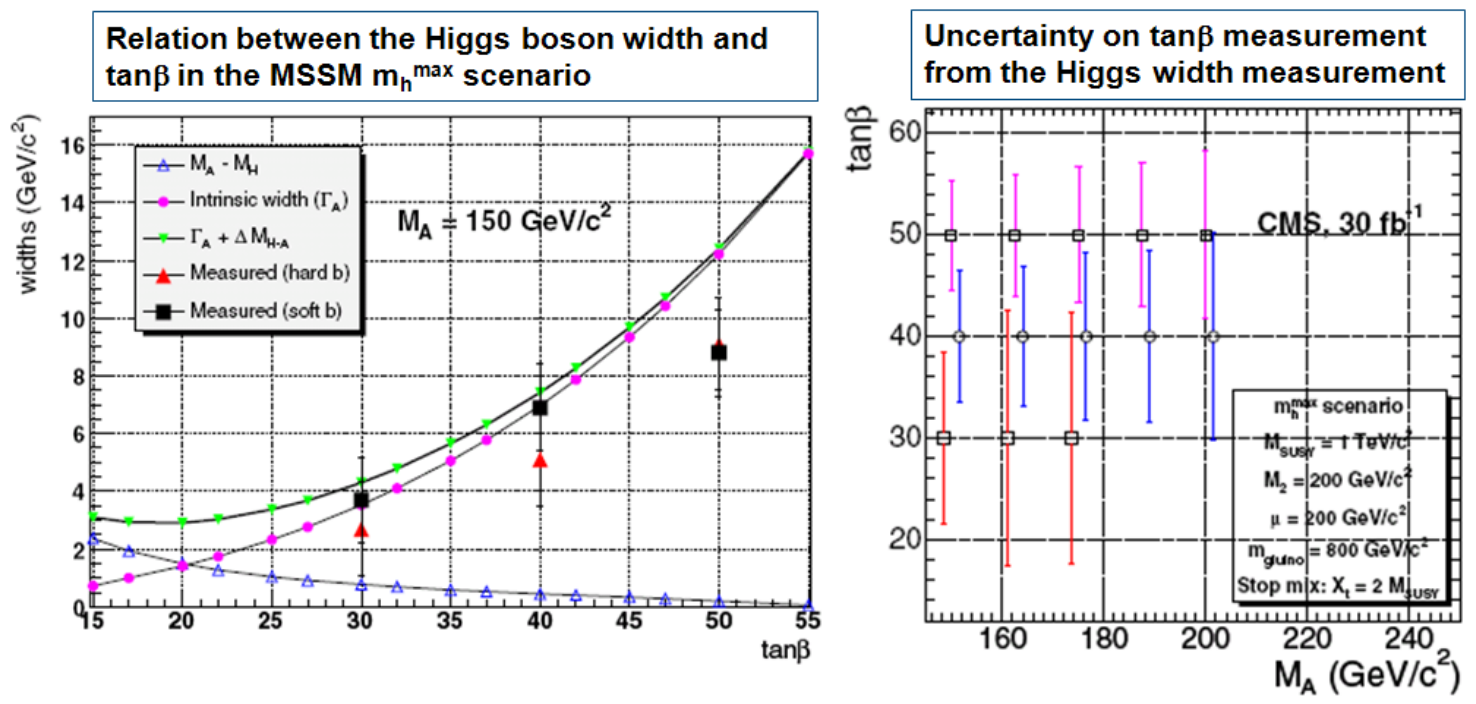

Figure 7: Determination of $\tan \beta$ from the measurement of the Higgs width [12]. Left: comparison of the intrinsic Higgs width (shown as solid circles) with the measured values (solid triangles and solid squares) for $M_{A}=150 \mathrm{GeV}$. Fitting the measured Higgs mass distribution with a Voigt function, the contribution of the measured mass resolution is subtracted. The mass difference $M_{A}-M_{H}$ is also plotted as a function of $\tan \beta$ (open triangles). For low $\tan \beta$, the mass difference is larger than the intrinsic Higgs width. Thus the measured width is the sum of the intrinsic width and of Higgs mass difference (inverted triangles): $\Gamma_{A}+\left(M_{H}-M_{A}\right)$. Right: expected measured values of $\tan \beta$ with their uncertainty as a function of $M_{A}$ obtained by exploiting the MSSM relation between the Higgs boson width and $\tan \beta$ in the $m_{h}^{\max }$ scenario. A theoretical uncertainty of $15 \%$ is included.

expected branching ratio $\left(\sim 10^{4}\right)$ the precise measurement of the dimuon mass provides an excellent opportunity to suppress the $t \bar{t}$ and $\mu \mu b \bar{b}$ backgrounds. The associated Higgs boson production with $\mathrm{b}$ quarks is exploited to suppress the Drell-Yan dimuon background using b-tagging. Figure 7 shows the expectation for the $\tan \beta$ measurement with its uncertainty as a function of $M_{A}$, derived using the MSSM relation between the Higgs boson width and $\tan \beta$. The $\tan \beta$ parameter can be further constrained using the cross section measurement and exploiting the $\tan \beta$ dependence, $\sigma B \sim(\tan \beta)^{2}$.

Another final state with great potential for detailed investigation is the four charged lepton final state. In the SM, the $H \rightarrow Z Z$ branching ratio is expected to be between $20 \%$ and $30 \%$ for $M_{H} \gtrsim 2 m_{Z}$. In the MSSM this decay may be significantly suppressed. Thus, for $M_{H} \gtrsim 2 m_{Z}$, if a substantial Higgs decay branching ratio into four lepton is measured, the observed Higgs signal is likely not a MSSM Higgs boson. At the same time, if the measured Higgs mass is substantially beyond the upper limit from SM electroweak fits, the observed signal is also unlikely to be a SM Higgs boson, as BSM physics must be advocated to explain the unexpectedly large Higgs mass. Thus, the discovery of a Higgs-like particle with large mass and large $H \rightarrow Z Z$ branching ratio can be a smoking gun for BSM physics, other than the MSSM, that will require further investigation about the nature of the observed four lepton resonance. In this respect, the measurements of the spin and CP quantum numbers of the observed Higgs-like particle are especially interesting. It is 

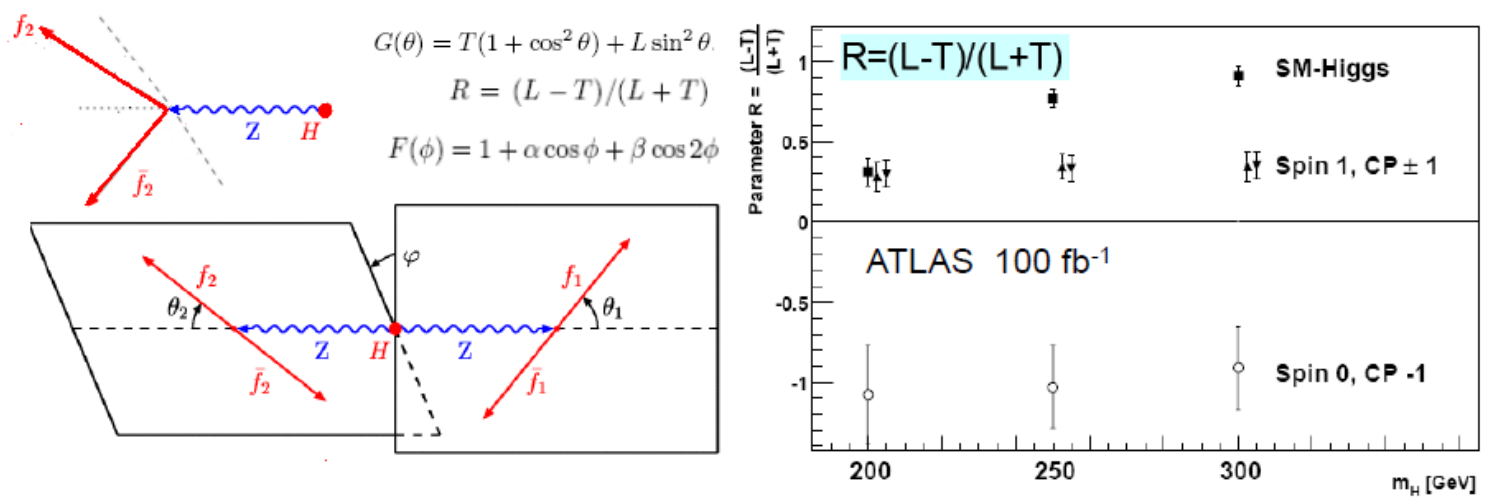

Figure 8: Determination of Higgs spin and CP quantum numbers. Left: definition of the angle $\theta$ [16] between the momentum of the charged lepton and the direction of the receding Higgs boson in the corresponding $\mathrm{Z}$ boson rest frame and of the angle $\phi$ between the two decay planes of the $\mathrm{Z}$ bosons from the Higgs boson decay. The definitions of the functions $\mathrm{G}(\theta)$ and $\mathrm{F}(\phi)$ Right: expected measured values of the parameter R as a function of the Higgs mass [17] for different spin and CP value hypotheses. The uncertainties are shown for an integrated luminosity of $100 \mathrm{fb}^{-1}$.

possible to extract information about the spin and $\mathrm{CP}$ quantum numbers of the Higgs boson from the angular correlations in the $\mathrm{H} \rightarrow \mathrm{ZZ} \rightarrow 4 \ell$ channel [16]. Three main variables are used to study the spin and $\mathrm{CP}$ of the Higgs boson in the $\mathrm{H} \rightarrow \mathrm{ZZ} \rightarrow 4 \ell$ channel: $\mathrm{R}, \alpha$ and $\beta$. $\mathrm{R}$ is given by $\mathrm{R}=(\mathrm{L}$ $-\mathrm{T}) /(\mathrm{L}+\mathrm{T})$, where $\mathrm{L}$ and $\mathrm{T}$ are the number of longitudinally and transversely polarized $\mathrm{Z}$ bosons in the $\mathrm{H} \rightarrow \mathrm{ZZ} \rightarrow 4 \ell$ decay, respectively. The values for $\mathrm{L}$ and $\mathrm{T}$ are obtained from fitting the the function $\mathrm{G}(\theta)$ ( Figure 8) to the measured distribution of the angle $\theta$ between the momentum of the charged lepton and the direction of the receding Higgs boson in the corresponding $\mathrm{Z}$ boson rest frame. The parameters $\alpha$ and $\beta$ are obtained from fitting the function $\mathrm{F}(\phi)$ (Figure 8 ) to the distribution of the angle $\phi$ between the two decay planes of the $\mathrm{Z}$ bosons in the Higgs boson rest frame. As and example, the expected measure values for R are shown in Figure 8 [17] for the SM Higgs boson and for three other combinations of spin and CP: spin-0 CP odd, spin-1 CP even and spin-1 CP odd. For Higgs masses above $200 \mathrm{GeV}$, and integrated luminosity of $100 \mathrm{fb}^{-1}$, a SM Higgs-like particle can be disentangled from other hypothesis with more than $5 \sigma$ signal significance.

\section{Conclusions}

The detailed simulation studies performed by the LHC collaborations show that, at the design center-of-mass energy of $14 \mathrm{TeV}$ and with adequate integrated luminosity (between one to few tens of $\mathrm{fb}^{-1}$ ) the LHC experiments will explore a large variety of scenarios beyond the SM where the existence of Higgs bosons will be proven or excluded. The discovery of Higgs-like signals will raise fundamental questions as to the nature of these particles and the underlying BSM physics. These questions will be addressed by measuring precisely Higgs masses, spin, CP numbers and couplings. In combination with the BSM Higgs discovery, other BSM signals may be detected and provide further information to investigate the nature of the underlying BSM physics, such that the actual 
discovery and measurements capabilities of the LHC experiments may encompass the predictions. As of today, one fact is certain: the excellent performance of the LHC detectors with the first highenergy collision data, delivered by the LHC in the 2009 run, show that the experiments are ready for early precision measurements of the SM as well as for discoveries of expected or unexpected signals beyond the SM.

\section{Acknowledgments}

I wish to thank the organizers for their warm hospitality and the inspiring atmosphere of this Symposium. I am indebted with my colleagues of the ATLAS and CMS collaborations and I am particularly grateful to K.A. Assamagan, M. Grünewald, A. Koryotov, C. Mariotti and W. Murray for their suggestions and constructive criticism in the preparation of this talk.

\section{References}

[1] See e.g. F. Cerutti and S. Desai. Contributions to these Proceedings.

[2] T. Hambye and K. Riesselmann, Phys. Rev. D 55, 7255 (1997).

[3] ALEPH, DELPHI, L3, OPAL Collaborations and LEP Higgs Working Group, Phys. Lett. B 565, 61 (2003).

[4] ALEPH, CDF, D0, DELPHI, L3, OPAL, SLD Collaborations, LEP Electroweak, Tevatron Electroweak, SLD Electroweak and Heavy Flavour Working Groups, CERN-PH-EP/2009-023, Nov. 2009, http://lepewwg.web.cern.ch/LEPEWWG/stanmod/summer2009/s09_ew.pdf.

[5] L. Randall and R. Sundrum, Phys. Rev. Lett. 83, 3370 (1999).

[6] N. Arkani-Hamed, A.G. Cohen, E. Katz and A.E. Nelson, JHEP 0207:034 (2002).

[7] R. Contino, Contribution to these Proceedings.

[8] LHC, ALICE, ATLAS, CMS, LHCB Collaborations, JINST 0803, S08001-7 (2008).

[9] M. S. Carena and H. E. Haber, Prog. Part. Nucl. Phys. 50, 63 (2003) and references therein.

[10] G. Davies, Contribution to these Proceedings.

[11] ATLAS Collaboration, "Expected Performance of the ATLAS Experiment - Detector, Trigger and Physics", arXiv:0901.0512v4 [hep-ex].

[12] CMS Collaboration, J. Phys. G34, 995 (2007).

[13] CMS Collaboration, "Projected sensitivity for Higgs boson searches for $7 \mathrm{TeV}$ and $1 \mathrm{fb}^{-1}$ ", https://twiki.cern.ch/twiki/bin/view/CMS/PublicPhysicsResultsHIGSevenTeV, March 2010.

[14] ALEPH, DELPHI, L3 and OPAL Collaborations and LEP Higgs Working Group, Eur. Phys. J. C47, 547 (2006).

[15] S. Bansal, Contribution to these Proceedings.

[16] S. Y. Choi et al., Phys. Lett. B 553, 61 (2003).

[17] C. P. Buszello et al.,, Eur. Phys. J. C32, 209 (2004). 